\title{
Comparing B3LYP and B97 dispersion-corrected functionals for studying adsorption and vibrational spectra in nitrogen reduction
}

\author{
Esther Grossman ${ }^{1,1}$, Damilola Daramola ${ }^{1}$, and Gerardine Botte ${ }^{2}$ \\ ${ }^{1}$ Ohio University \\ ${ }^{2}$ Texas Tech University
}

May 12, 2020

\begin{abstract}
Electrochemical ammonia synthesis is being actively studied as a low temperature, low pressure alternative to the Haber-Bosch process. This work studied iridium as the electrochemical catalyst, following a previous study of adsorption characteristics on platinum. The characteristics studied include bond energies, bond lengths, spin densities, and free and adsorbed vibrational frequencies for the molecules N2, N, NH, NH2, and NH3. Overall, these descriptive characteristics explore the use of dispersioncorrected Density Functional Theory methods that can model N2 adsorption - the key reactant for electrochemical ammonia synthesis via transition metal catalysis. Specifically, three methods were tested: hybrid B3LYP, a dispersion-corrected form B3LYP-D3, and semi-empirical B97-D3. The latter semi-empirical method was explored to increase the accuracy obtained in vibrational analysis as well as reduce computational time. Two lattice surfaces, (111) and (100), were compared. The adsorption energies are stronger on (100) and follow the trend EB3LYP > EB3LYP-D3 > EB97-D3 on both surfaces.
\end{abstract}

Comparing B3LYP and B97 dispersion-corrected functionals for studying adsorption and vibrational spectra in nitrogen reduction

Esther F. Grossman, 11Esther F. GrossmanDepartment of Physics and Astronomy, Center for Electrochemical Engineering Research, Ohio University, Athens, Ohio 45701, United StatesPresent Address: Jacobs School of Engineering, UC San Diego, La Jolla, California 92093, United States Damilola A. Daramola, 22Damilola A. DaramolaDepartment of Chemical and Biomolecular Engineering, Center for Electrochemical Engineering Research, Ohio University, Athens, Ohio 45701, United States and Gerardine G. Botte 33 Gerardine G. BottePresent Address: Department of Chemical Engineering, Texas Tech University, Lubbock, Texas 79409, United States

Correspondence to: Gerardine G. Botte (E-mail:gerri.botte@ttu.edu )

\section{Introduction}

In 1954, about 2,900,000 metric tons of ammonia were produced in the United States, $70 \%$ of which was utilized by the agricultural sector. ${ }^{1}$ More than 60 years later, in 2018, U.S. ammonia production reached an estimated 12,500,000 metric tons, with the world total coming in at 140,000,000 tons. ${ }^{2}$ This essential industrial process currently uses over $1 \%$ of the

world's power. ${ }^{3}$ Ammonia is used in the synthesis of a variety of products, most notably, fertilizers. The Haber-Bosch process, the process by which ammonia is produced, is a large source of carbon emissions primarily because the synthesis reaction is performed at very high temperatures and pressures (350-5500C and 150-350 atm). ${ }^{4}$ Researchers are looking for alternative mechanistic pathways to bypass the energy requirements of Haber-Bosch. Such processes include biological nitrogen fixation, ${ }^{5}$ ultraviolet promotion of $\mathrm{N}_{2},{ }^{6}$ and electrochemical nitrogen reduction. ${ }^{7-11}$ For the industrial Haber-Bosch process, iron and ruthenium 
are commonly studied ${ }^{12-14}$ because transition metals readily adsorb and release oxygen. ${ }^{15}$ Catalysts studied for the electrochemical process include ruthenium, iron oxides, and nitride compounds. ${ }^{7,9,10,16}$ Osmium and platinum have also been identified as good catalysts. ${ }^{17,18}$ Experimentally, Allagui et al. found that a bimetallic PtIr nanoparticle catalyst decomposed ammonia at a $33 \%$ higher rate than platinum alone. ${ }^{19}$ Le Vot et al. found that iridium lowers the overpotential of ammonia oxidation. ${ }^{20}$ Boggs and Botte came to the same conclusion in their study of PtIr anodes deposited on carbon substrates in alkaline solution. ${ }^{21}$ Lastly, Estejab and Botte used small cluster calculations to show $\mathrm{Ir}_{3}$ is more active than $\mathrm{Pt}_{3}$ in nitrogen oxidation. ${ }^{18}$ These studies suggest a basis for exploring mechanistic behavior on larger clusters of iridium for nitrogen reduction. By better understanding the thermodynamics of the reaction, new steps can be taken in the lab to increase ammonia yield.

When using clusters to represent catalysts, the computational scheme is very important. The popular B3LYP Density Functional Theory (DFT) method does not describe dispersion between molecules well and thus cannot appropriately describe $\mathrm{N}_{2}$ adsorption on a catalyst surface. Hopmann et al. computationally studied bond formation and ligand exchange reactions mediated by iridium in solution. They found that the free energies of B3LYP systems with dispersion provide moderate overall accuracy and significant improvement over B3LYP alone. ${ }^{22}$ Therefore, this study tests B3LYP against its dispersion-corrected form, as well as against a less computationally expensive dispersion-corrected functional, B97-D3.

This paper has two primary objectives. The first is to calculate adsorption energies, binding sites, and vibrational spectra for the intermediates involved in the ammonia synthesis reaction on pure iridium. This data can be used to shed light on the reaction mechanism of ammonia synthesis on the (111) and (100) surfaces. The activation energies, which correlate to the speeds of the possible reactions, can also be calculated, but they are beyond the scope of this study. Calculations on pure iridium will serve as a direct comparison to previous calculations performed on pure platinum clusters. ${ }^{23}$ Key differences will be analyzed with respect to adsorption energetics and bonding sites. These calculations can also serve as a benchmark for future bimetallic calculations. Additionally, significant energy savings would result from an electrochemical process that takes place at ambient temperatures and pressures. ${ }^{24,25}$ Thus, all calculations in this study are run at standard temperature and pressure (STP). The second objective is to compare the accuracy of three Density Functional Theory methods - B3LYP, B3LYP-D3 and B97-D3 - in predicting bonding sites, bonding lengths, bonding energies and vibrational frequencies on the catalyst surface. These characteristics are well-modeled by cluster calculations. ${ }^{18,23,26}$

\section{Methods}

All calculations were implemented in Gaussian 09 using default convergence limits. ${ }^{27}$ The computational schemes compared in this study are B3LYP, B3LYP-D3, and B97-D3. B3LYP is the Becke-style 3-Parameter functional. ${ }^{28,29}$ The dispersion corrected version, B3LYP-D3, uses Grimme's dispersion and the Beck-Johnson damping parameter. ${ }^{30}$ B97-D3 is Grimme's functional with Becke-Johnson damped dispersion as well. ${ }^{29-31}$ While B3LYP is a hybrid functional, often used to achieve bond lengths and vibrational frequencies close to experimental values, ${ }^{32}$ B97 is a semi-empirical pure functional which delivers speedier results on larger clusters of atoms.

For consistency between methods and with previous calculations on platinum, ${ }^{23}$ we will use the LANL2DZ basis set for the iridium atoms and $6-311++\mathrm{g}^{* *}$ basis sets for nitrogen and hydrogen. ${ }^{23}$

The (111) and (100) surfaces of the face-centered cubic iridium catalyst are both modeled in this study. As a default in Gaussian 09, the most abundant isotope (Ir-193) is used for calculations. ${ }^{33}$ The clusters built for these calculations are kept at frozen coordinates during optimization, with an atomic Ir-Ir spacing of $0.3839 \mathrm{~nm} .{ }^{34}$ All adsorbates are unfrozen. Frequency calculations are conducted at the minimum energy geometries obtained from optimization. Bending modes with an IR intensity less than $20 \times 10^{-40} \mathrm{esu}^{2} \mathrm{~cm}^{2}$ or Raman activity less than $20 \mathrm{~A}^{4} / \mathrm{AMU}$ are considered difficult to observe against the cluster vibrations and are therefore not all recorded in this paper. For general comparisons, this paper classifies vibrations as weak (activity [?] 40), moderate (activity $=40-100)$, strong (activity $=100-200)$, and very strong $($ activity $=200+$ ). 


\section{Cluster Geometry}

A cluster size of $15 \mathrm{Ir}$ atoms was chosen by analyzing the cohesive energy and spin density of $\operatorname{Ir}_{\mathrm{n}}$ clusters $(\mathrm{n}=10,15,20,25,30)$ shown in Figure 1. Each Ir atom has nine valence electrons spread amongst its $5 \mathrm{~d}$ and $6 \mathrm{~s}$ orbitals. An initial estimate for the number of unpaired electrons in our modeled catalyst was determined using Kua and Goddard's Interstitial Electron Model. ${ }^{35}$ Their model estimates the unpaired electrons for the (111) surface and hypothesizes that the surface has $\mathrm{s}^{1} \mathrm{~d}^{8}$ configuration. This methodology was used to describe the (100) surface as well, as an initial guess. The number of unpaired electrons in the ground state and the energies of each cluster are calculated and presented in Table 1. Cluster energies were calculated for two multiplicities above and below the ground state multiplicity to evaluate convergence to a minimum. Clusters larger than 20 atoms did not converge using B3LYP or B3LYP-D3. Only the (100) surface of B97-D3 converged for clusters larger than 25. As shown in Table 1, the larger the cluster, the closer the cohesive energy comes to approaching the experimental bulk cohesive energy of iridium, 670 $\mathrm{kJ} / \mathrm{mol} .{ }^{36,37}$ (111) surfaces are more tightly packed and thus approach the bulk cohesive energy quicker than (100) surfaces. A cluster size of 15 atoms is ultimately chosen over a size of 20 atoms because the spin densities across the 15-atom cluster are closer to the number of unpaired electrons per atom in the ground state d-orbital i.e. two (see Figure 2).

The 15-atom cluster is chosen for both the (111) and (100) surfaces; they are pictured in Figure 1c and 1d respectively. They are both two-layer clusters with enough surface area that all principal adsorption sites on the surface are distanced from the catalyst's edge, reducing "edge effects."

\section{Simulation Procedure}

First, the molecules $\mathrm{N}_{2}, \mathrm{~N}, \mathrm{NH}, \mathrm{NH}_{2}$, and $\mathrm{NH}_{3}$ are optimized in the gas-phase using each of the three DFT functionals. The optimized molecules are then placed on the (100) and (111) catalyst clusters at one of the preferential sites in Figure 3. Each molecule is optimized separately at every possible preferential site in order to determine the lowest energy position on the catalyst. For $\mathrm{N}_{2}$ optimizations, that includes testing end-on and side-on $\mathrm{N}-\mathrm{N}$ configurations at each site. After optimization, frequency calculations are run on the lowest energy adsorption sites. This data is then compared between functionals and to other theoretical and experimental benchmarks.

\section{Results}

Binding energy is calculated from equation 1:

$E_{\text {binding }}=E_{I r+\mathrm{NH}_{x}}-E_{\mathrm{Ir}}-E_{\mathrm{NH}_{x}}(1)$

where binding energies are based on electronic energies for calculated preferential adsorption sites, and on zero-point and thermal-corrected energies elsewhere.

\section{Preferential Adsorption Sites}

The molecules successfully adsorb to the surface of each catalyst at the sites show in Figure 4. About one hundred structures were successfully optimized, with nine failures that were due to an inability to converge or bond to the catalyst surface. When two different starting geometries converged to the same final site, the lower energy optimization was used in calculations for Figure 4.

$\operatorname{Ir}_{15}$ (111) Cluster

$\mathrm{N}$ and $\mathrm{NH}$ adsorb at top, hexagonal close-packed, and face-centered cubic positions using all methods B3LYP, B3LYP-D3, and B97-D3. The HCP position is most favored, closely followed by FCC. This is very similar to the theoretical findings of Krekelberg et al., whose study used periodic DFT methods PW91 and RPBE. ${ }^{38}$ They found that HCP and FCC were equally favorable. $\mathrm{NH}_{2}$ adsorbs at the bridge position for all methods and the top position for B3LYP-D3 and B97-D3, with the bridge position being slightly more favorable. $\mathrm{NH}_{3}$ also adsorbs at top and bridge positions for all methods, and the top position is most favorable. This agrees with Krekelberg's theoretical findings, but is in conflict with an experiment that 
found adsorption of $\mathrm{NH}_{3}$ at $\mathrm{FCC} / \mathrm{HCP} .{ }^{38}$ Although for most of the molecules in this study, more than one adsorption site is found possible, none of the methods could find a minimum at the HCP or FCC position for $\mathrm{NH}_{3}$.

$\operatorname{Ir}_{15}$ (100) Cluster

$\mathrm{N}$ adsorbs at the top and bridge positions for all methods and for B97-D3, the hollow position as well; however, the bridge position is the most favorable across all methods. For NH, B3LYP-D3 does not converge to a bridge site, but B3LYP and B97-D3 do. B97-D3 shows an additional, less favorable adsorption at the top position. Both B3LYP and B3LYP-D3 converged to hollow sites located only at the edges of the surface. Due to edge effects on this hollow site, it is likely that bridge is the most favorable site for NH. This is in agreement with adsorption of monatomic $\mathrm{O}$ on $\operatorname{Ir}(100)$, which also has two unpaired electrons and adsorbs at the bridge position. ${ }^{39} \mathrm{NH}_{2}$ and $\mathrm{NH}_{3}$ have only one favorable site - bridge and top respectively.

Overall, all methods yield the same preferential adsorption sites on both the (100) and (111) surfaces, with the exception of NH on (100). B3LYP always gives the smallest binding energies, while its dispersion-corrected form gives larger binding energies and B97-D3 gives the largest binding energies.

$\mathrm{N}_{2}$

$\mathrm{N}_{2}$ comparisons are limited to B3LYP-D3 and B97-D3. B3LYP simulations do not have the dispersion calculations necessary to adsorb N-N to the catalyst, as DFT cannot accurately describe the types of dispersion forces that would exist between $\mathrm{N}_{2}$ and the metal surfaces. ${ }^{31}$ Therefore, no $\mathrm{N}_{2}$ simulations converged with B3LYP. The binding energies calculated for the other methods are shown in Figure 5 with respect to the angle of adsorption on the catalyst surface ( $\mathrm{N}-\mathrm{N}$ bond with Ir surface). B97-D3 suggests that the end-on configuration is favorable for both (111) and (100) surfaces. This position hovers at the top site, in agreement with Krekelberg. ${ }^{38}$ B3LYP-D3 suggests that there are multiple orientations of $\mathrm{N}_{2}$ within $0.05 \mathrm{eV}$ that successfully adsorb, with the lowest energy configurations being mostly parallel to the surface (side-on) for both (111) and (100).

\section{Frequency Calculations and Bonding Characteristics}

The most favored adsorption site for each of the molecules in Figure 4 - also illustrated in Figure 6 - is used for a frequency calculation.

\section{Adsorption of $\mathrm{N}_{2}$}

Diatomic nitrogen adsorbed on the two surfaces is pictured in Figure $6 \mathrm{e}$ and $6 \mathrm{j}$. This end-on, top position corresponds to the lowest energy optimization achieved with B97-D3. The N-N bond makes an angle of 890 with the (111) and (100) surfaces. The B3LYP-D3 N-N bonds make angles of 300 and 70 with the (111) and (100) surfaces respectively. The spin density per atom before and after adsorption of $\mathrm{N}_{2}$ can be found in Supplementary Data Figure S1. With B3LYP-D3, the spin density profile is consistent before and after adsorption, with a slight reduction in spin on atom 5 for the (111) surface because the N-N bond is angled 300 towards that atom, providing context for slight charge transfer between the $\mathrm{N}$ and Ir atom. With B97-D3, the (111) surface sees a similar effect. Notably, there is a greater variance in B97-D3 ground state spin density before and after bonding than with B3LYP-D3. On the (100) surface, the spin density drop is particularly steep $\left(\mathrm{N}_{2}\right.$ oriented 890$)$. This suggests bonding of the inner $\mathrm{N}$ atom to $\mathrm{Ir}$ atom number 5 . This bond results from an interaction between the Ir $5 \mathrm{~d}$-states and $\pi$-bonds polarized in the direction of Ir, thus weakening the N-N bond and laying ground for N-Ir electron pairing. Adsorbate energy is still relatively small due to $\sigma$-bond repulsion along the axis of the bond. ${ }^{43}$ Covalent bond formation is also evidenced by the bond lengths in Table 2a, where $\mathrm{N}_{2}$ molecules that adsorbed preferentially at the top position, perpendicular to the catalyst (B97-D3), adsorbed greater than $1 \mathrm{~nm}$ closer than those adsorbed at an angled or horizontal position (B3LYP-D3). Bond energies are all far from the experimentally determined $9.8 \mathrm{eV}$, but similar to theoretical predictions of -0.54 and $-0.21 \mathrm{eV} .{ }^{38}$

Vibrational analysis of $\mathrm{N}_{2}$ as a free molecule is broken down by method in Table $2 b{ }^{38,40,41}$ B97-D3 pre- 
dicts the stretching mode of $\mathrm{N}_{2}$ to within $10 \mathrm{~cm}^{-1}$, while both B3LYP methods largely overestimate it. After adsorption, B3LYP-D3 predicts a 10 $\mathrm{cm}^{-1}$ decrease in the frequency of the stretching mode, while B97-D3 predicts an $\sim 170 \mathrm{~cm}^{-1}$ decrease in the frequency. Experiment gives the adsorbed frequency to be $2185 / 2210 / 2223$ $\mathrm{cm}^{-1}$, which is right on par with B97-D3 predictions. ${ }^{38,41}$ From this we can conclude B97-D3 correctly predicts the lowest energy position on the surface is at the top position, with the $\mathrm{N}_{2}$ molecule perpendicular to the surface.

\section{Adsorption of $N$}

Monatomic nitrogen is pictured in Figure 6a and $6 \mathrm{f}$ at its preferential positions - HCP (111) and bridge (100). Average spin density before and after adsorption of $\mathrm{N}$ on the cluster surface can be found in Supplementary Data Figure S2. All methods show a consistent spin distribution on non-bonding atoms before and after binding on the (111) surface. Atoms 2, 5, and 6 are the clear sites of bonding for the three unpaired electrons of nitrogen because the spin density drops by approximately one at each location. On the (100) surface, bonding is evident on only atoms 1 and 5 . The third unpaired electron on $\mathrm{N}$ is not clearly bound to any particular Ir atom.

Bond length as described in Table $3 \mathrm{a}$ is the shortest distance between the $\mathrm{N}$ atom and the catalyst surface. Bond length for B3LYP and its dispersion-corrected form are 1.17/1.16 $\AA$ and $1.26 \AA$ on the (111) and (100) surfaces respectively; B97-D3 length is slightly longer - 1.22/1.29 A. These are close to that computed by Krekelberg - $1.16 \AA$ and $1.11 \AA .{ }^{38}$ Moving from B3LYP to B3LYP-D3 to B97-D3, bond energy gets progressively stronger.

Table $3 \mathrm{~b}$ gives the IR and Raman frequencies for various vibrational modes. ${ }^{38}$ The asymmetric stretch on the (100) surface is very strongly Raman active, while the symmetric stretch is moderately Raman and IR active. All vibrations on the (111) surface are weak.

\section{Adsorption of $\mathrm{NH}$}

Figure $6 \mathrm{~b}$ and $6 \mathrm{~g}$ shows NH adsorbed at its most preferential site - HCP (111) and bridge (100). Supplementary Data Figure S3 shows the average spin density before and after adsorption of NH on the cluster surface. On the (111) surface, the nitrogen atom is adsorbed between atoms 2, 5, and 6, just as lone $\mathrm{N}$ is. The spin density per atom takes on an almost identical shape with B3LYP, B3LYP-D3, and B97-D3 (at a lower average spin density for the latter). For the (100) surface, B3LYP-D3 converges to only an erroneous edge-effect hollow position. For the other two methods, the two unpaired $\mathrm{e}^{-}$of $\mathrm{NH}$ form bonds with bridging Ir atoms 5 and 8. The spin structure of B97-D3 is noticeably more similar to B3LYP after adsorption than before adsorption.

Bond length in Table 4a is consistent between the three methods for (111) - 1.23/1.24 A. On the (100) surface, B97-D3 is $0.07 \AA$ shorter. As with each of the other molecules, bond energy follows the trend $\mathrm{E}_{\mathrm{B} 3 \mathrm{LYP}}>$ $\mathrm{E}_{\mathrm{B} 3 \mathrm{LYP}-\mathrm{D} 3}>\mathrm{E}_{\mathrm{B} 97-\mathrm{D} 3}$.

Table $4 \mathrm{~b}$ shows NH frequencies for gas phase and Ir-adsorbed states and how these frequencies compare to benchmark experimental work. ${ }^{44,45}$ The $\mathrm{N}-\mathrm{H}$ stretch perpendicular to the catalyst surface is strongly Raman active on both surfaces. On the (100) surface, the wagging mode is strongly Raman active as well, according B97-D3 calculations. This same mode under the B3LYP method is only moderately Raman active with a stronger IR activity. All other modes are weak.

\section{Adsorption of $\mathrm{NH}_{2}$}

Figure $6 \mathrm{c}$ and $6 \mathrm{~h}$ shows $\mathrm{NH}_{2}$ adsorbed at its preferential site - bridge. Supplementary Data Figure S4 shows the average spin density before and after adsorption of $\mathrm{NH}_{2}$ to the surface. On the (111) surface, the adsorption takes place on Ir atoms 5 and 6 as evidenced by the drop in spin density on those atoms. On the (100) surface, bonding takes place on atoms 1 and 5 (B3LYP and B97-D3), and 5 and 8 (B3LYP-D3). Again, the spin distribution per atom for each method looks nearly identical after bonding. 
Bond lengths in Table 5a are similar between methods, varying by at most $0.04 \AA . \mathrm{E}_{\mathrm{B} 3 \mathrm{LYP}}>\mathrm{E}_{\mathrm{B} 3 \mathrm{LYP}-\mathrm{D} 3}>$ $\mathrm{E}_{\mathrm{B} 97-\mathrm{D} 3}$ for both surfaces.

Table 5b lists the vibrational modes of gas-phase and adsorbed $\mathrm{NH}_{2} \cdot{ }^{44,46,47}$ B3LYP and its dispersioncorrected form match experiment for the symmetric mode slightly better than B97-D3, while B97-D3 matches the asymmetric mode best. On both the (111) and (100) surface, the scissoring mode is IR active, while the symmetric stretch is strong and the antisymmetric stretch is weak in Raman activity. The stretching mode between $\mathrm{N}$ and the catalyst surface itself is very weak on both surfaces.

\section{Adsorption of $\mathrm{NH}_{3}$}

Figure 6d and $6 \mathrm{i}$ show $\mathrm{NH}_{3}$ adsorbed at its most preferential site - top. Supplementary Data Figure S5 gives the spin density of the catalyst before and after bonding. On the (111) and (100) surfaces, ammonia adsorbs on Ir atom 5 (a top position atom). The spin density drops by $\sim 0.6-1$ unpaired electron at that site (the same magnitude as the drop in density for $\mathrm{NH}_{2}$ ), indicating $\mathrm{NH}_{3}$ may be covalently bonding with the top Ir atom. However, the adsorption energy is still much lower than for $\mathrm{NH}_{2}$.

Table 6a gives bond lengths and energies of ammonia's adsorption. Bond lengths are similar on the (111) surface. On the (100) surface, the bond length with B3LYP is $0.12 \AA$ longer than with B3LYP-D3, whose bond length is $0.12 \AA$ longer than with B97-D3. The trend in binding energy is $\mathrm{E}_{\mathrm{B} 3 \mathrm{LYP}}>\mathrm{E}_{\mathrm{B} 3 \mathrm{LYP}-\mathrm{D} 3}>$ $\mathrm{E}_{\mathrm{B} 97-\mathrm{D} 3}$.

Table $6 \mathrm{~b}$ lists the principal vibrational modes of $\mathrm{NH}_{3}$ in gas-phase and Ir-adsorbed phase. ${ }^{48-50}$ For all gasphase vibrations, all three methods overestimate the experimental benchmarks. B97-D3 comes closest to experiment for both of the stretching modes and the bending mode, significantly reducing the error involved in ignoring anharmonic frequencies during calculations. ${ }^{23}$ After adsorption the stretch vibrations are redshifted $\sim 30 \mathrm{~cm}^{-1}$, and the wag is blue-shifted $\sim 160-200 \mathrm{~cm}^{-1}$. Other theoretical work comes closest to B97-D3 predictions for all except the weak linear stretch between $\mathrm{NH}_{3}$ and the surface, for which B3LYP comes closest. $^{38}$ The wag is a strong IR mode. The symmetric modes are strongly Raman active (very strong on the B3LYP (100) cluster) and all other modes are weakly Raman active.

\section{Conclusions}

This study had two objectives. The first was to calculate local adsorption characteristics - binding sites, bond distances, adsorption energies, and vibrational spectra for select intermediates involved in the ammonia synthesis reaction on pure iridium. These intermediates are $\mathrm{N}_{2}, \mathrm{~N}, \mathrm{NH}, \mathrm{NH}_{2}$, and $\mathrm{NH}_{3}$. Secondly, three DFT methods - B3LYP, B3LYP-D3, and B97-D3 - were compared to determine the effect dispersion has on the popular B3LYP method and contrast it with a faster semi-empirical GGA, B97-D3.

\section{B3LYP vs. B3LYP-D3 (effect of dispersion)}

The dispersive function's primary effect is to account for long-range forces in molecular adsorption on catalyst surfaces by the inclusion of van der Waals forces, as evidenced in Figure 4. Using Grimme's dispersion with B3LYP amounts to an increase in binding energy of about $0.5 \mathrm{eV}$ for several of the intermediates. This not only brings the energy closer to experimental benchmarks but is necessary for the primary reactant in ammonia synthesis, $\mathrm{N}_{2}$, to be adsorbed on the modeled catalyst. Without it, DFT optimizations do not converge and thus cannot be included in a mechanistic study involving $\mathrm{N}_{2}$ as a reactant.

Aside from energetic differences and very small decreases in bond length, the dispersion-corrected B3LYP has a very similar electronic structure as B3LYP. Both methods predict the same ground state spin multiplicity and very similar spin density profiles on the (111) and (100) surfaces before and after adsorption of intermediates. This is consistent with Grimme's claim that the dispersive term does not rely on nor affect electronic structure. ${ }^{51}$ Select vibrational modes of adsorbed molecules are affected by dispersion, but most are not. Unadsorbed molecular vibrational modes are not affected at all.

B3LYP-D3 vs. B97-D3 (effect of hybrid and semi-local GGAs) 
B97-D3 serves to increase the binding strength of the system and of adsorbates more than B3LYP-D3 because GGA bonded interactions tend to be stronger than their hybrid counterparts that include exact exchange. ${ }^{52}$ This paper shows a decrease in adsorption energy from B3LYP-D3 to B97-D3 of $\sim 0.2 \mathrm{eV}$. Bond lengths with B97-D3 are slightly smaller for $\mathrm{N}_{2}, \mathrm{NH}, \mathrm{NH}_{2}$, and $\mathrm{NH}_{3}$, and larger for $\mathrm{N}$. Gas-phase vibrational frequencies with B97-D3 are closer to experimental benchmarks as well as theoretical benchmarks of non-empirical GGAs. B3LYP-D3 overestimates the frequency of most modes, sometimes by as much as $100-200 \mathrm{~cm}^{-1}$, as in the case of the $\mathrm{N}_{2}$ stretch. Adsorbed to the catalyst, B97-D3 is the only method that correctly predicts the lowest energy bonding pattern and orientation of $\mathrm{N}_{2} \cdot{ }^{43,53}$ Despite both functionals including dispersion corrections, both continue to underbind dispersion-bound $\mathrm{N}_{2}$.

A notable difference in the ground state multiplicity and spin density profile of the catalyst surface exists between B97-D3 and the hybrid methods (figures in Supplementary Data). Despite this fact, after adsorption of an intermediate to the catalyst surface, the resulting spin density profiles follow an almost identical trend as the hybrid methods. This may imply B97-D3 has more difficulty predicting the correct ground state structure before adsorption since the surface is a charge-transfer complex with unpaired electrons, two characteristics often modeled better by methods with exact exchange (although dispersion helps). ${ }^{52}$ Since the density profiles are a match after adsorption, all three methods result in the same site preferences across all adsorbates (with the exception of $\mathrm{N}_{2}$ and $\mathrm{NH}(100)$ using B3LYP-D3). This fact, combined with more accurate thermodynamic data and reduced computation time, make B97-D3 a viable option for continued study of this system and related metal cluster systems. B97-D3 includes minimal empiricism, sitting in the top 10 least-parameterized semi-empirical methods, according to one study of seventy functionals. ${ }^{52}$

\section{(111) vs. (100) (effect of lattice plane)}

Adsorbates on the (100) surface are more strongly bonded than on the (111) surface, except for $\mathrm{NH}_{3}$, where the bonding energies are comparable. The hollow site on (100) is not a preferred binding site for any of the intermediates. The spacing between Ir atoms is too great in this region for a minimum energy to be found in the overlap between the orbitals from $\mathrm{N}$ and those from the four Ir nearest neighbors in the hollow position. As a result, $\mathrm{N}$ and $\mathrm{NH}$ adsorb at the bridge position on the (100) surface (forming two bonds each), whereas they adsorb at an FCC site on (111) (forming three bonds and two bonds respectively). Despite the increased binding energy, Ir-adsorbate bond lengths are greater on the (100) surface, except for $\mathrm{NH}_{2}$, where they are slightly shorter. Frequency calculations result in a ${ }^{\sim} 5-150 \mathrm{~cm}^{-1}$ difference between vibrations of the same mode on different lattice planes.

\section{Platinum vs. Iridium (effect of catalyst)}

Previous calculations using B3LYP on a 15-atom (111) cluster of platinum are directly comparable to the B3LYP results in this study. ${ }^{23}$

In the ground state of the platinum cluster, there are 12 unpaired electrons. In this study, there are 27, in addition to there being a higher cohesive energy of Ir. Preferential catalyst positions are a close match, the only difference being this study shows a small preference for adsorption of $\mathrm{N}$ and $\mathrm{NH}$ at the $\mathrm{HCP}$ site over the FCC site.

Binding strength increases with the same trend $\mathrm{NH}_{3}<\mathrm{NH}_{2}<\mathrm{NH}<\mathrm{N}$ as on iridium. By removing the thermal corrections from this study's binding energies, they directly compare to the electronic binding energies calculated in the platinum study. The results are as follows: $\mathrm{N}$ adsorption is $33 \mathrm{~kJ} / \mathrm{mol}$ stronger, $\mathrm{NH}$ is 40 $\mathrm{kJ} / \mathrm{mol}$ stronger, $\mathrm{NH}_{2}$ is $26 \mathrm{~kJ} / \mathrm{mol}$ stronger, and $\mathrm{NH}_{3}$ is $4 \mathrm{~kJ} / \mathrm{mol}$ weaker on iridium than on platinum. The weaker $\mathrm{Ir}-\mathrm{NH}_{3}$ bond is interesting because platinum shows a reduction in spin density of only 0.2 , suggesting a non-covalent dipole interaction, while on iridium the spin density decreases by $\sim 1.2$, suggesting a stronger, covalent interaction. Additionally, the shorter bond length of adsorbed $\mathrm{NH}_{3}(2.23 \AA$ on platinum and $2.17 \AA$ on iridium) would seemingly indicate a stronger $\mathrm{Ir}-\mathrm{NH}_{3}$ bond. For comparison, bond lengths for $\mathrm{NH}_{2}$ are $\sim 0.01 \AA$ longer, for $\mathrm{NH}$ are $\sim 0.03 \AA$ longer, and for $\mathrm{N}$ are $\sim 0.04 \AA$ longer on Ir than on Pt.

\section{Future Work}


In future work, it would be beneficial to move forward with a single DFT method and perform calculations for $\mathrm{N}_{2} \mathrm{H}, \mathrm{N}_{2} \mathrm{H}_{2}, \mathrm{~N}_{2} \mathrm{H}_{3}$, and $\mathrm{N}_{2} \mathrm{H}_{4}$, to evaluate the dissociative and associative pathways for the electrochemical ammonia synthesis process, applying a voltage and taking coverage effects into account. Experimental followup could be performed with vibrational analysis.

\section{Acknowledgments}

This research was supported by the Ohio Supercomputer Center under grant number OSC-PHS0269, the Ohio University Honors Tutorial College, and the Center for Electrochemical Engineering Research.

\section{References and Notes}

1. B. S. Duff, Petroleum Processing 1955, 10 , 223-228.

2. L. E. Apodaca, Mineral commodity summaries 2019: U.S. Geological Survey 2019 , 116-117.

3. M. Kitano, Y. Inoue, Y. Yamazaki, F. Hayashi, S. Kanbara, S. Matsuishi, T. Yokoyama, S.-W. Kim, M. Hara, H. Hosono, Nature Chemistry 2012 , 4 , 934-940.

4. R. R. Schrock, Proceedings of the National Academy of Sciences2006 , 103, 17087-17087.

5. M. G. Scheibel, S. Schneider, Angewandte Chemie International Edition 2012 , 51 , 4529-4531.

6. Y. Lu, Y. Yang, T. Zhang, Z. Ge, H. Chang, P. Xiao, Y. Xie, L. Hua, Q. Li, H. Li, B. Ma, N. Guan, Y. Ma, Y. Chen, ACS Nano2016 , 10 , 10507-10515.

7. Y. Abghoui, E. Skúlason, Catalysis Today 2017 ,286 , 78-84.

8. S. Back, Y. Jung, Phys. Chem. Chem. Phys. 2016 ,18, 9161-9166.

9. K. Kim, C.-Y. Yoo, J.-N. Kim, H. C. Yoon, J.-I. Han, Korean Journal of Chemical Engineering 2016 , 33 , $1777-1780$.

10. F. Kosaka, N. Noda, T. Nakamura, J. Otomo, Journal of Materials Science 2017 , 52 , 2825-2835.

11. E. Skúlason, T. Bligaard, S. Gudmundsdóttir, F. Studt, J. Rossmeisl, F. Abild-Pedersen, T. Vegge, H. Jónsson, J. K. Nørskov, Phys. Chem. Chem. Phys. 2012, 14, 1235-1245.

12. W. Arabczyk, U. Narkiewicz, K. Kalucki, Vacuum 1994 ,45 , 267-269.

13. W. Arabczyk, U. Narkiewicz, D. Moszynski, Langmuir 1999 , 15 , 5785-5789.

14. G. Buzzi Ferraris, G. Donati, F. Rejna, S. Carrà, Chemical Engineering Science 1974, 29 , 1621-1627.

15. Chemical Age 1920 , 3 , 56-59.

16. Y. Abghoui, E. Skúlasson, Procedia Computer Science2015 , 51, 1897-1906.

17. C. J. H. Jacobsen, S. Dahl, B. S. Clausen, S. Bahn, A. Logadottir, J. K. Nørskov, Journal of the American Chemical Society2001, 123, 8404-8405.

18. A. Estejab, G. G. Botte, Computational and Theoretical Chemistry 2016 , 1091 , 31-40.

19. A. Allagui, M. Oudah, X. Tuaev, S. Ntais, F. Almomani, E. A. Baranova, International Journal of Hydrogen Energy 2013 ,38 , 2455-2463.

20. S. Le Vot, L. Roué, D. Bélanger, Journal of Power Sources2013, 223 , 221-231.

21. B. K. Boggs, G. G. Botte, Electrochimica Acta 2010 ,55 , 5287-5293.

22. K. H. Hopmann, Organometallics 2016 , 35 , 3795-3807.

23. D. A. Daramola, G. G. Botte, Computational and Theoretical Chemistry 2012, 989 , 7-17.

24. R. Lan, J. T. S. Irvine, S. Tao, Scientific Reports2013 , 3: 1145 , 1-7. 
25. B. L. Sheets, G. G. Botte, Chemical Communications2018, 54 , 4250-4253.

26. D. A. Daramola, D. Singh, G. G. Botte, J. Phys. Chem. A2010 , $114,11513-11521$.

27. M. J. Frisch, G. W. Trucks, H. B. Schlegel, G. E. Scuseria, M. A. Robb, J. R. Cheeseman, G. Scalmani, V. Barone, B. Mennucci, G. A. Petersson, H. Nakatsuji, M. Caricato, X. Li, H. P. Hratchian, A. F. Izmaylov, J. Bloino, G. Zheng, J. L. Sonnenberg, M. Hada, M. Ehara, K. Toyota, R. Fukuda, J. Hasegawa, M. Ishida, T. Nakajima, Y. Honda, O. Kitao, H. Nakai, T. Vreven, J. A. Montgomery, Jr., J. E. Peralta, F. Ogliaro, M. Bearpark, J. J. Heyd, E. Brothers, K. N. Kudin, V. N. Staroverov, T. Keith, R. Kobayashi, J. Normand, K. Raghavachari, A. Rendell, J. C. Burant, S. S. Iyengar, J. Tomasi, M. Cossi, N. Rega, J. M. Millam, M. Klene, J. E. Knox, J. B. Cross, V. Bakken, C. Adamo, J. Jaramillo, R. Gomperts, R. E. Stratmann, O. Yazyev, A. J. Austin, R. Cammi, C. Pomelli, J. W. Ochterski, R. L. Martin, K. Morokuma, V. G. Zakrzewski, G. A. Voth, P. Salvador, J. J. Dannenberg, S. Dapprich, A. D. Daniels, O. Farkas, J. B. Foresman, J. V. Ortiz, J. Cioslowski, and D. J. Fox, Gaussian 09, Revision E.01, Gaussian, Inc.: Wallingford, CT, 2013.

28. A. D. Becke, The Journal of Chemical Physics 1993,98, 5648-5652.

29. Gaussian, Inc., Density Functional (DFT) Methods. http://gaussian.com/dft/ (accessed April 26, 2018).

30. S. Grimme, S. Ehrlich, L. Goerigk, Journal of Computational Chemistry 2011, 32 , 1456-1465.

31. S. Grimme, Journal of Computational Chemistry 2006 ,27 , 1787-1799.

32. J. P. Perdew, M. Ernzerhof, K. Burke, The Journal of chemical physics 1996 , 105 , 9982-9985.

33. C. J. Cramer, In Essentials of Computational Chemistry: Theories and Models; John Wiley \& Sons: Hoboken, NJ, 2004 ; Chapter 10, pp 355-383.

34. H. Gao, Y. Xiong, X. Liu, D. Zhao, Y. Feng, L. Wang, J. Wang, Applied Surface Science 2016 , 389 , 211-215.

35. J. Kua, W. A. Goddard, The Journal of Physical Chemistry B1998 , 102 , 9481-9491.

36. KnowledgeDoor LLC, Cohesive Energy. http://www.knowledgedoor.com/2/elements_handbook/cohesive_energy.html (accessed January 25, 2018).

37. C. Kittel, In Introduction to Solid State Physics; John Wiley \& Sons: Hoboken, NJ, 2005 ; pp 50.

38. W. P. Krekelberg, J. Greeley, M. Mavrikakis, The Journal of Physical Chemistry B 2004, 108, 987-994.

39. K. Johnson, Q. Ge, S. Titmuss, D. A. King, J. Chem. Phys.2000 , 112 , 8.

40. K. P. Huber; G. Herzberg, In Molecular Spectra and Molecular Structure; Van Nostrand Reinhold Company: New York, NY, 1979 ; Vol. 4, pp 420.

41. A. Ravi, D. A. King, N. Sheppard, Transactions of the Faraday Society 1968 , 64, 3358-3360.

42. J. C. L. Cornish, N. R. Avery, Surface Science 1990 ,235 , 209-216.

43. A. Nilsson, L. G. M. Pettersson, J. K. Nørskov, In Chemical Bonding at Surfaces and Interfaces; Elsevier: Amsterdam; Boston, Mass.; Oxford, UK, 2008 ; Chapter 2, pp. 79-90.

44. D. E. Milligan, M. E. Jacox, Journal of Chemical Physics1965 , 43 , 4487-4493.

45. R. S. Ram, P. F. Bernath, K. H. Hinkle, The Journal of chemical physics 1999 , 110 , 5557-5563.

46. T. Amano, P. F. Bernath, A. R. W. McKellar, Journal of Molecular Spectroscopy 1982, 94, 100-113.

47. J. B. Burkholder, C. J. Howard, A. R. W. McKellar, Journal of Molecular Spectroscopy 1988 , 127 , $415-424$.

48. K. Nakamoto, In Infrared and Raman Spectra of Inorganic and Coordination Compounds; Wiley: New York, NY, 1978 . 
49. T. Shimanouchi, In Tables of Molecular Vibrational Frequencies; United States Department of Commerce: Washington, D.C., 1972 ; V. 1, Chapter 4, pp. 15.

50. J. P. Contour, G. Pannetier, Journal of Catalysis1972 , 24 , 434-445.

51. S. Grimme, J. Antony, S. Ehrlich, H. Krieg, The Journal of Chemical Physics 2010 , 132 , 154104.

52. N. Mardirossian, M. Head-Gordon, Molecular Physics2017 , 115 , 2315-2372.

53. C.-C. Wang, S. S. Siao, J.-C. Jiang, The Journal of Physical Chemistry C 2010, 114 , 18588-18593.

\section{Table \& Figure Captions}

Table 1 . Cohesive energy of iridium clusters with $n$ atoms, where $n=10,15,20,25$, and 30 . The ground state spin multiplicity was predicted using the Interstitial Electron model and verified by testing two multiplicities above and below the ground state. The lowest energy state is presented here for each cluster size.

Table 2a. Bond energies and bond lengths are given for the most favorable orientation of $\mathrm{N}_{2}$ on the (111) and (100) surfaces.

Table 2b. Vibrational modes and their corresponding frequencies (in $\mathrm{cm}^{-1}$ ) for free and adsorbed $\mathrm{N}_{2}$ on both the (111) and (100) surface. Values are compared to existing experimental and theoretical observation. ${ }^{\mathrm{a}}$ experimental; ${ }^{\mathrm{b}}$ theoretical; ${ }^{\mathrm{c}} \mathrm{IR} ;{ }^{\mathrm{d}}$ EELS; $*$ index plane not specified

Table 3a. Bond energies and bond lengths are given for $\mathrm{N}$ at its lowest energy position - HCP for the (111) surface and bridge for the (100) surface.

Table 3b. Vibrational modes and their corresponding frequencies (in $\mathrm{cm}^{-1}$ ) for adsorbed $\mathrm{N}$ at its lowest energy position - HCP for the (111) surface and bridge for the (100) surface. The displacement of N for each stretching mode is labeled as either perpendicular or parallel to the cluster surface. Values are compared to existing experimental and theoretical observation.

${ }^{a}$ theoretical - RPBE

Table 4a. Bond energies and bond lengths are given for NH at its lowest energy position - HCP for the (111) surface and bridge for the (100) surface.

Table 4b. Vibrational modes and their corresponding frequencies $\left(\right.$ in $\left.\mathrm{cm}^{-1}\right)$ for free and adsorbed $\mathrm{NH}$ at its lowest energy position - HCP for the (111) surface and bridge for the (100) surface. The displacement of N in Ir-N the stretches is labeled as either perpendicular or parallel to the cluster surface. Values are compared to existing experimental and theoretical observation.

${ }^{\mathrm{a}}$ experimental; $\mathrm{IR}$; F-failed to converge

Table 5a. Bond energies and bond lengths are given for $\mathrm{NH}_{2}$ at its lowest energy position - bridge for both the (111) surface and (100) surface.

Table 5b. Vibrational modes and their corresponding frequencies $\left(\mathrm{in} \mathrm{cm}^{-1}\right.$ ) for free and adsorbed $\mathrm{NH}_{2}$ at its lowest energy position - bridge for the (111) and (100) surface. Values are compared to existing experimental and theoretical observation. Asymmetric and symmetric stretches are abbreviated as a. stretch and s. stretch.

${ }^{\mathrm{a}}$ experimental; ${ }^{\mathrm{b}} \mathrm{IR} ;{ }^{\mathrm{c}}$ Difference Frequency Laser Spectroscopy

Table 6a. Bond energies and bond lengths are given for $\mathrm{NH}_{3}$ at its lowest energy position - top for both the (111) and (100) surface.

Table 6b. Vibrational modes and their corresponding frequencies (in $\mathrm{cm}^{-1}$ ) for free and adsorbed $\mathrm{NH}_{3}$ at its lowest energy position - top for the (111) and (100) surface. Values are compared to existing experimental and theoretical observation. Asymmetric and symmetric stretches are abbreviated as a. stretch and s. stretch.

${ }^{\mathrm{a}}$ experimental; ${ }^{\mathrm{b}}$ theoretical; ${ }^{\mathrm{c}} \mathrm{IR} ;{ }^{*}$ index plane not specified 
Figure 1. $\operatorname{Ir}_{\mathrm{n}}$ clusters modelled, where $\mathrm{n}=10,15,20,25$, and 30 . The left column depicts the (111) surfaces and the right column depicts the (100) surfaces. Atoms are numbered so they can be matched with their respective spin densities in Figure 2.

Figure 2. Spin density of $\operatorname{Ir}_{\mathrm{n}}$ clusters $(\mathrm{n}=10,15,20,25)$. Columns 1, 2, and 3 show the cluster spin densities using B3LYP, B3LYP-D3, and B97-D3 respectively. The 30-atom cluster only converged for the (100) surface at the B97-D3 level of theory, so it was not included in this comparison.

Figure 3. Preferential sites for adsorption on the (111) (above) and (100) (below) $\operatorname{Ir}_{15}$ surfaces. B=Bridge, $\mathrm{T}=$ Top, $\mathrm{HCP}=$ Hexagonal Close-Packed, FCC=Face-Centered Cubic, and $\mathrm{H}=$ Hollow.

Figure 4. Binding energy (based on electronic energies) of molecules adsorbed on the (111)[a-d] and (100)[e-h] surfaces using the three different functionals. $\mathrm{B}=$ Bridge, $\mathrm{T}=\mathrm{Top}, \mathrm{HCP}=$ Hexagonal Close-Packed, FCC=FaceCentered Cubic, and $\mathrm{H}=$ Hollow.

Figure 5. Binding energy (based on electronic energies) of $\mathrm{N}_{2}$ adsorbed on the (111) and (100) surfaces at various angles. $\Theta$ is the angle the $\mathrm{N}-\mathrm{N}$ bond makes with the catalyst surface.

Figure 6. Top view of preferred adsorption sites for adsorbed $\mathrm{N}, \mathrm{NH}, \mathrm{NH}_{2}$, and $\mathrm{NH}_{3}$ on the (111)[a-d] and (100)[f-i] surface. A side view is given for $\mathrm{N}_{2}$ [e and j]. Images shown are from the B97-D3 optimizations, but site preferences are consistent across all methods. However, the orientation of $\mathrm{N}_{2}$ is different using B3LYP-D3.

\section{GRAPHICAL ABSTRACT}
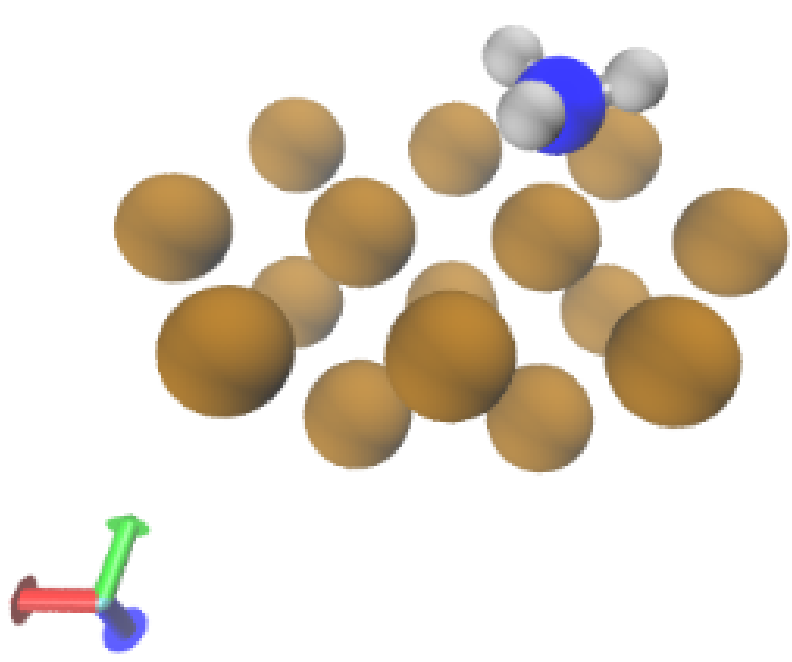

AUTHOR NAMES: Esther F. Grossman, Damilola A. Daramola, and Gerardine G. Botte 
TITLE: Comparing B3LYP and B97 dispersion-corrected functionals for studying adsorption and vibrational spectra in nitrogen reduction

TEXT: Increasing the yield of ammonia in the electrochemical synthesis process gets researchers one step closer to replacing the energy-intensive Haber-Bosch process. Benchtop experiments with iridium have been shown to produce good ammonia yields via the electrochemical process. The present study investigates which Density Functional Theory methods best characterize the molecules involved in catalysis over iridium and provides theoretical benchmarks that can be used to understand the underlying mechanism.

\section{Hosted file}

Figure1.docx available at https://authorea.com/users/287843/articles/450303-comparingb3lyp-and-b97-dispersion-corrected-functionals-for-studying-adsorption-and-vibrationalspectra-in-nitrogen-reduction

\section{Hosted file}

Figure2.docx available at https://authorea.com/users/287843/articles/450303-comparingb3lyp-and-b97-dispersion-corrected-functionals-for-studying-adsorption-and-vibrationalspectra-in-nitrogen-reduction

\section{Hosted file}

Figure3.docx available at https://authorea.com/users/287843/articles/450303-comparingb3lyp-and-b97-dispersion-corrected-functionals-for-studying-adsorption-and-vibrationalspectra-in-nitrogen-reduction

\section{Hosted file}

Figure4.docx available at https://authorea.com/users/287843/articles/450303-comparingb3lyp-and-b97-dispersion-corrected-functionals-for-studying-adsorption-and-vibrationalspectra-in-nitrogen-reduction

\section{Hosted file}

Figure5.docx available at https://authorea.com/users/287843/articles/450303-comparingb3lyp-and-b97-dispersion-corrected-functionals-for-studying-adsorption-and-vibrationalspectra-in-nitrogen-reduction

\section{Hosted file}

Figure6.docx available at https://authorea.com/users/287843/articles/450303-comparingb3lyp-and-b97-dispersion-corrected-functionals-for-studying-adsorption-and-vibrationalspectra-in-nitrogen-reduction 